\title{
Educación formal y acciones de agrupación y memoria en adultos: Un estudio microgenético *
}

\author{
MANUEL L. DE LA MATA \\ JOSÉ A. SÁNCHEZ \\ Universidad de Sevilla
}

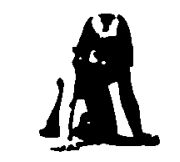

Resumen

Se estudió la influencia de la educación formal en el empleo de acciones de agrupación y memoria en sujetos adultos. El trabajo tenía una doble perspectiva, transversal y microgenética. Los sujetos pertenecian a tres niveles educativos y se les estudió a lo largo de cuatro dias, en dos pruebas de memorización y recuerdo y una prueba de categorización dirigida intercalada entre las anteriores. Los resultados mostraron la influencia esperada de la escolarización en el empleo de acciones de agrupación y memoria así como cambios a lo largo de las sesiones en el grupo intermedio. La interpretación de los resultados se basó en las nociones de subordinación de acciones mentales y de descontextualización de los instrumentos de mediación.

Palabras clave: Memoria, Agrupación, Acciones, Educación formal, Educación de adultos, Descontextualización de los instrumentos de mediación.

\section{Formal education, categorization and memory actions in adults: A microgenetic study}

\section{Abstract}

The influence of formal education on the acquisition and use of categorization and memory in adults was studied. The work had a double cross-sectional and microgenetic perspective. Subjects came from three educational levels and were studied across four days, in two memory tasks and in a directed categorization task intercalated between them. Results showed the expected influence of schooling over categorization and memory, as well as microgenetic changes across sessions. We interpreted results in terms of subordination of memory actions and decontextualization of mediational means.

Keywords: Memory, Clustering, Actions, Formal education, Adult education, Decontextualization of mediational means.

* Nota de los editores: Algunos de los datos presentados en este artículo son interpretados en el artículo de Ramírez, Cubero y Santamaría, que aparece en el número 51-52 de Infancia y Aprendizaje, por lo que es recomendable que ambos trabajos se lean como un conjunto.

Dirección del autor: Laboratorio de Actividad Humana. Universidad de Sevilla. Facultad de Psicología. Avda. San Francisco Javier s/n. 41005 Sevilla 


\section{INTRODUCCION}

El estudio de la influencia de los factores culturales en los procesos de pensamiento tiene una historia importante dentro de la psicología cognitiva. Ha dado lugar a un campo de investigación que se ha denominado psicología transcultural (Triandis y Heron, 1981). Dentro de este ámbito general de problemas, uno de los más estudiados ha sido el de la influencia de diferentes variables culturales en la memoria. El objetivo de nuestro trabajo es presentar una investigación dedicada al estudio de esta cuestión. Específicamente, se ha investigado la influencia de una variable cultural, como es la escolarización, en el uso y adquisición de estrategias o acciones de memorización.

En la psicología transcultural existe una amplia tradición de estudios sobre la memoria. Estas investigaciones han establecido diferencias ligadas al nivel de escolarización en el uso de destrezas de memoria (Brown, 1977; Rogoff, 1981; Cole y Scribner, 1974; Scribner y Cole, 1981; Rogoff y Mistry, 1985; Sharp, Cole y Lave, 1979; Wagner, 1981). Se ha puesto de manifiesto que los individuos con experiencia escolar suficiente tienden a obtener mejores resultados en tareas que exigen el empleo de destrezas de indole muy fina y flexible. Si bien es necesario reconocer las dificultades de interpretación asociadas a las investigaciones que comparan sujetos con diferente experiencia cultural (ver De la Mata y Ramírez, 1989, para una revisión más detallada de la cuestión), es evidente, no obstante, que los resultados de la investigación cultural sobre la memoria nos indican que la escolarización promueve un tipo de destrezas que son detectadas en las pruebas experimentales habituales. Estas destrezas están ligadas a unos contextos socioculturales determinados, característicos de las sociedades modernas. En estos términos interpretamos los resultados de la investigación cultural y los de nuestro propio trabajo.

El estudio de la influencia de la escolarización en adultos permite observar el efecto de cambios y factores culturales al margen de los procesos de desarrollo madurativo. La educación de adultos ofrece un campo privilegiado para el análisis de la influencia de los factores culturales en el desarrollo de las funciones mentales superiores. Podemos afirmar que se trata de un auténtico "laboratorio natural" en el que el investigador sociocultural puede observar procesos de adquisición y desarrollo cognitivo culturalmente determinados. Brinda, por ello, una oportunidad para superar algunos de los problemas metodológicos que la investigación trascultural ha llevado aparejada (Rogoff, 1981) y, sobre todo, la ocasión de analizar la proyección de diferentes actividades socioculturales en procesos de pensamiento como los que se han estudiado en este trabajo.

Una buena parte de las investigaciones que se han centrado en la influencia de la cultura sobre la memoria ha adoptado el concepto de estrategia como unidad de estudio de la memoria. El concepto de estrategia fue formulado por primera vez por Flavell (1970) y se ha convertido en una noción clave para estudiar la adquisición y el desarrollo de las habilidades de memoria. Una estrategia se define como una acción dirigida a un objetivo de memoria. En su definición, es fundamental la naturaleza instrumental, la relación medios/fin. Recientemente, el grupo de investigación de S. G. Paris de la Universidad de Michigan ha ofrecido una conceptualiza- 
ción más completa de las estrategias de memoria. Según estos autores (Paris, Lipson y Wixon, 1983; Paris, Newman y Jacobs, 1985), lo que define a una estrategia no es la forma, el tipo de conducta implicada, sino la función, es decir, la relación medios/fin percibida por el sujeto y el uso intencional que hace éste de los procedimientos instrumentales de memorización. Estos autores establecen, además, las características básicas de las estrategias de memoria.

En primer lugar, afirman la naturaleza personalizada de las estrategias. Una estrategia ha de ser construida por el sujeto o, en caso de que la emplee bajo la dirección de otra persona, debe asumir la relación instrumental implicada. En la medida en que ha de ser significativa para el sujeto que la emplea, su uso está estrechamente vinculado a los contextos de desarrollo y actividad. Como señala Rogoff (1982), el contexto de cualquier actividad cognitiva incluye a la tarea que se está desarrollando, los materiales implicados, la situación social y el objetivo de dicha actividad. Estos elementos configuran la propia actividad y, en este caso, definen a las estrategias mismas. La naturaleza contextual de las estrategias nos remite a su determinación social. Las estrategias se adquieren y emplean en un medio social. Este medio social tiene una doble dimensión. En su dimensión microsocial, las estrategias se adquieren en las interacciones cara a cara entre individuos. En sus relaciones con los padres, maestros o compañeros, el niño va adquiriendo y perfeccionando medios apropiados para resolver problemas de memoria. Como señalan Paris, et al. (1985), no se trata de afirmar que todas las estrategias se adquieren en la instrucción directa, sino que la relación con otras personas suministra oportunidades para su descubrimiento, al modelar la conducta eficaz e incrementar las demandas de uso autocontrolado de éstas. En estas «zonas de desarrollo próximo", los padres, profesores o compañeros guían al niño en la adquisición de estrategias y promueven la transferencia de la responsabilidad de seleccionar medios y fines apropiados. Las interacciones cara a cara ocurren además en el contexto de unas instituciones y de unas prácticas culturales que definen, por una parte, los medios y fines de memorización apropiados, y, por otra, la actuación de los participantes. Pese al indudable interés de esta cuestión, existen muy pocos estudios de la influencia de las interacciones próximas en la adquisición y desarrollo de destrezas de memoria. Los trabajos de Rogoff (Rogoff y Gardner, 1984) representan una de las pocas excepciones en este sentido. Los planos macrosocial y microsocial a los que se refieren Paris y su grupo, no se superponen uno a otro sin más, sino que están estrechamente conectados. Por una parte, la ocurrencia y las funciones que cumplen los participantes en las interacciones cara a cara están definidas por la cultura. La cultura organiza la ocurrencia de zonas de desarrollo próximo en las que se produce la transferencia de las destrezas culturales y cognitivas (LCHC, 1983; Cole, 1984). Por otra parte, las prácticas y las instituciones culturales mismas sólo existen en la medida en que son recreadas por individuos concretos en sus interacciones diarias.

Otra característica importante de las estrategias se relaciona con el desarrollo en el tiempo. Según Paris, las estrategias tienen un carácter temporalizado, que remite a la idea de desarrollo. En este sentido, no sólo interesa el desarrollo ontogenético, el más estudiado tradicionalmente, sino también otras formas como son el desarrollo cultural y el desarrollo mi- 
crogenético, a las que hemos prestado especial atención. Existen pocos trabajos que aborden el estudio de estrategias de memoria con una perspectiva metodológica de índole microgenética, a pesar de su indudable interés. El trabajo de Paris, Newman, Kelly y McVey (1982) es una excepción. Estos autores muestran la importante función que cumplen factores de naturaleza metacognitiva, específicamente, el aprendizaje de la significación y la utilidad de las estrategias, en su adquisición y dominio.

La concepción de Paris sobre las estrategias de memoria es un desarrollo del enfoque vygotskiano en este ámbito. Por ello, resulta de gran interés para avanzar en el estudio de la memoria dentro de un enfoque sociocultural. Uno de los autores (De la Mata, 1988) ha propuesto una integración de las investigaciones soviéticas y occidentales sobre el desarrollo de las habilidades de memoria. Mantenemos la preferencia por el término acción, de tradición soviética (Leontiev, 1973-74, 1981; Wertsch, 1985a), sobre el de estrategia, por tener el primero un carácter más definido como unidad de estudio de los procesos mentales. En todo caso, la utilización que hace Paris del término estrategia es plenamente coherente con el concepto de acción, adoptado como unidad de análisis de las funciones mentales por el enfoque sociocultural (Wertsch, 1985a).

Una de las acciones (o estrategias, en la terminología occidental) más estudiadas, tanto en la investigación evolutiva como cultural, ha sido la organización del material y, más específicamente, la agrupación de éste en categorías de diversa índole. En relación a la investigación cultural, se ha constatado que la experiencia escolar va asociada al uso de criterios de agrupación taxonómica. El uso de este tipo de criterio se refleja en los resultados obtenidos, en los que el número de elementos recordados suele relacionarse directamente con la experiencia escolar de los sujetos. Al mismo tiempo, el análisis de la organización del recuerdo revela una organización basada en agrupaciones categoriales taxonómicas.

Los primeros trabajos de Cole y cols. (Cole, et al., 1971) mostraron que los niños norteamericanos alcanzaban una ejecución general mejor que los niños del grupo kpelle de Liberia. Los niños norteamericanos comenzaron, desde los diez años, a agrupar los estímulos por categorías semánticas tras sucesivos ensayos. En los kpelle, por el contrario, no aparecieron apenas agrupaciones de este tipo. Los resultados reseñados se repitieron básicamente con sujetos mayas mexicanos (Sharp, Cole y Lave, 1979), y con sujetos vais de Liberia (Scribner y Cole, 1981). En el primero de estos estudios se encontró mejor recuerdo y agrupación en los sujetos mayas y mestizos con prolongada experiencia escolar (hasta la enseñanza secundaria). El trabajo de Zivian y Darjes (1983) pone de manifiesto diferencias semejantes entre sujetos adultos de cultura occidental con distinto grado de experiencia escolar. Los sujetos que acudían a la escuela (estudiantes universitarios jóvenes y de mediana edad) recordaron y agruparon más estímulos que los individuos de edad mediana o avanzada cuya experiencia escolar era más breve y estaba alejada en el tiempo.

En relación a los resultados reseñados, hay que tener en cuenta que la escolarización suministra abundantes oportunidades de trabajar de este modo, con materiales a los que el sujeto tiene que imponer una organización explícita de manera deliberada, sin que la propia estructura de estos materiales ni las condiciones de la tarea suministren un apoyo claro de cómo 
organizarlos. La agrupación categorial de materiales que no poseen una organización interna clara, por tanto, parece estar estrechamente relacionada con la experiencia escolar.

Las diferencias culturales en el recuerdo desaparecen, sin embargo, cuando se utilizan materiales estructurados, instrucciones que favorecen el establecimiento de relaciones entre los estímulos y, en general cuando las condiciones de realización de las pruebas facilitan la organización interna del material (Brown, 1977; Cole y Scribner, 1974, 1977; Mandler et al., 1980; Paris, Newman y Jacobs, 1985; Rogoff y Mistry, 1985). Scribner (1974) relata un experimento llevado a cabo con sujetos kpelle en el que la presentación de estímulos agrupables se hacía acompañada de un objeto real que funcionaba como clave. En este experimento, la presentación de claves verbales no mejóró el recuerdo ni la agrupación en ninguna de las condiciones, excepto cuando se instaba a los sujetos a recordar los estímulos sistemáticamente, categoría por categoría. Estos sujetos mantuvieron buenos resultados en un ensayo adicional. Por el contrario, los resultados descendieron marcadamente en el ensayo final en las condiciones en que no se suministraba la ayuda descrita. Parece, por tanto, que es posible mejorar el recuerdo y la agrupación de estos sujetos mediante la instrucción y el entrenamiento verbal explícito. Otro aspecto de las condiciones de realización de la tarea manipulado en la investigación de Scribner fue el permitir o no emplear las categorías propias de los kpelle frente a las consideradas semánticamente apropiadas por el experimentador. Los resultados de agrupación obtenidos por los sujetos mejoraron notablemente cuando se les permitió emplear sus propios criterios categoriales.

El estudio de las influencias sociales sobre la memoria se ha centrado, como hemos señalado, en la dimensión macrosocial, institucional, de las actividades de educación formal. Se ha establecido que estas actividades reúnen una serie de características que las diferencian de las de aprendizaje informal. Uno de estos rasgos básicos es su carácter descontextualizado (Greenfield, 1984; Greenfield y Lave, 1982; Scribner y Cole, 1973). El hecho de que el aprendizaje escolar, tal como se da actualmente en las sociedades modernas, tenga lugar en escenarios construidos culturalmente para este fin, separados de otros contextos cotidianos de actividad, conlleva el predominio de un «manejo» simbólico de la realidad. El lenguaje, especialmente un lenguaje de carácter descontextualizado, se convierte en el medio esencial de relación con la realidad extraescolar y de «manipulación» de ésta. El predominio del lenguaje impone la constante verbalización y explicación de las acciones realizadas. Otro rasgo importante es el hecho de que el aprendizaje se convierte en el motivo de la actividad (Wertsch, Minick y Arns, 1984). En la escuela, lo importante no es tanto resolver los problemas en sí mismos como aprender las reglas para resolverlos. La importancia del lenguaje y el énfasis en la adquisición de reglas generales imponen una constante verbalización y explicación de las acciones realizadas. En este sentido, algunos autores (Scribner y Cole, 1981; Tulviste, 1978, cit. en Wertsch, 1985a) han señalado que la influencia más persistente de la experiencia escolar sobre los procesos de pensamiento es la tendencia a explicar verbalmente los criterios y los pasos de resolución de las tareas. Desde un punto de vista sociocultural general, se puede afirmar que la educación formal promueve el desarrollo cultural de los procesos psicológicos al 
facilitar la descontextualización de los procedimientos de mediación de las acciones mentales (Luria, 1979; Wertsch, 1985a; Vygotsky, 1977, 1979). El término descontextualización se usa con sentidos diferentes en muchas ocasiones. Por otra parte, la consideración del desarrollo cultural como proceso de descontextualización parece contradecir la idea presentada más arriba de la actividad mental como algo ligado íntimamente al contexto. Siguiendo un enfoque sociocultural, es importante precisar que el avance en la línea de la descontextualización afecta a los procedimientos semióticos, no a las acciones en sí mismas. Si bien todas las acciones mentales, incluyendo las que se producen en el curso de las actividades de educación formal, están esencialmente ligadas al contexto, los procesos semióticos implicados pueden basarse en mayor o menor medida en el potencial contextualizador o descontextualizador del lenguaje (Wertsch, 1985b). Es en este terreno donde se produce el desarrollo hacia la descontextualización, no en las acciones mismas, que como tales se adquieren y se utilizan en contextos culturales específicos.

Como señalábamos más arriba, son escasos los trabajos que han adoptado una perspectiva microgenética para estudiar la adquisición y desarrollo de las acciones de memoria. El interés por este tipo de desarrollo, sin embargo, constituye una valiosa aportación del enfoque vygotskiano. Permite observar en pequeña escala, en períodos de tiempo muy breves, procesos de desarrollo que pueden ser en muchos sentidos semajantes a los que tienen lugar en el curso del desarrollo ontogenético, lo que ofrece la posibilidad de contemplar los aspectos fundamentales de dichos procesos de desarrollo con más claridad incluso que en la ontogénesis. Nuestro trabajo está diseñado para estudiar desarrollos de índole microgenética, observando las destrezas de memoria y los procedimientos semióticos de mediación en más de una sesión. Al adoptar una perspectiva microgenética, esperábamos determinar si el dominio de acciones de agrupación categorial facilitaría la utilización de dichas acciones al servicio de otro objetivo diferente, la memorización y el recuerdo. Específicamente, el objetivo era estudiar destrezas de agrupación categorial en dos planos diferentes: como acciones autónomas, en una tarea de emparejamiento categorial, y como acciones subordinadas, en una tarea de recuerdo de material agrupable, (ver De la Mata, 1988, para un desarrollo más extenso de los conceptos de acción autónoma y acción subordinada en tareas de memoria). La hipótesis de la que partíamos era que el dominio de la agrupación categorial en el plano de las acciones autónomas facilitaría la subordinación de éstas a otro objetivo más general, permitiendo su empleo como instrumento al servicio de la memorización y el recuerdo.

\section{METODO}

\section{Sujetos}

Participaron en la investigación 105 sujetos de centros del Programa de Educación de Adultos de Sevilla capital. Pertenecían a tres niveles educativos: alfabetización, pregraduado y graduado escolar ${ }^{1}$. La distribución por niveles fue homogénea, de modo que se estudiaron 35 sujetos de cada uno de ellos. 
Resulta interesante hacer referencia a la distribución por sexo de la muestra. De los 105 sujetos estudiados, $90(85,7 \%)$ fueron mujeres. Esta desproporción es algo menor en el grupo de graduado, donde las mujeres representaban únicamente el $65,7 \%$ de la muestra. Sin embargo, en los dos niveles más elementales, las mujeres representan el $98 \%$ del grupo. Esta desigualdad se debe a que la mayor parte de los alumnos de los centros de EPA, sobre todo en los niveles de alfabetización y pregraduado, son amas de casa de una edad comprendida entre los 45 y los 55 años. El grupo de graduado presenta unas características claramente diferenciadas de los demás niveles. La edad de estos alumnos suele oscilar entre los 18 y los 35 años y en su mayoría han tenido una experiencia escolar continuada, lo que no ocurre con los sujetos de alfabetización y pregraduado.

\section{Material}

- Trece láminas tamaño cuartilla (láminas de emparejamiento) en las que aparecían cuatro dibujos en cada una (Figura 1). El dibujo de la izquierda de la lámina estaba dentro de un recuadro. Se trataba en todos los casos de animales o plantas comunes - mamíferos o árboles respectivamente- . Junto a este dibujo había un recuadro en blanco. En la parte derecha de las láminas aparecían tres dibujos no enmarcados. Entre ellos había un dibujo de un ser vivo, animal o planta, un dibujo de un elemento que guardaba relación de tipo contextual con el ser vivo de la izquierda (pertenencia a situaciones cotidianas comunes: caballo-silla de montar, perro-caseta,burro-carro, etc.), y un tercer dibujo que representaba un elemento sin relación aparente con el de la izquierda. Las láminas estaban ordenadas en cinco niveles de complejidad, según la relación (más o menos evidente) entre el dibujo encuadrado y el ser vivo de la derecha.

- Dos juegos de 22 tarjetas (Figura 2). En cada tarjeta aparecía un dibujo de un elemento conocido. Las tarjetas eran agrupables según criterios categoriales (animales, plantas, prendas de vestir, instrumentos musicales, etc.) o contextuales (cigüeña-nido, toro-espada-sombrero, etc.).

\section{Procedimiento}

El estudio de los sujetos se realizó a lo largo de cuatro días, según la siguiente secuencia temporal:

Día 1. Primera prueba de memoria: Fase de estudio.

Día 2. Primera prueba de memoria: Fase de recuerdo.

Prueba de categorización: Fase de emparejamiento.

Día 3. Prueba de categorización: Fase de reconocimiento.

Segunda prueba de memoria: Fase de estudio.

Dí 4. Segunda prueba de memoria: Fase de recuerdo.

En las pruebas de memorización voluntaria se presentó a los sujetos una de las listas de material agrupable a la vez que se les informaba de que posteriormente tendrían que recordar el mayor número posible de los dibujos vistos. Se pidió a los sujetos que estudiaran el material con el fin de que pudiesen recordarlo, indicándoles que podían manipularlo de cualquier forma. Con la intención de inducir la emisión de habla se les comunicaba que podían hablar, diciéndoles que otros compañeros suyos lo hacían y eso les 
FIgURA 1
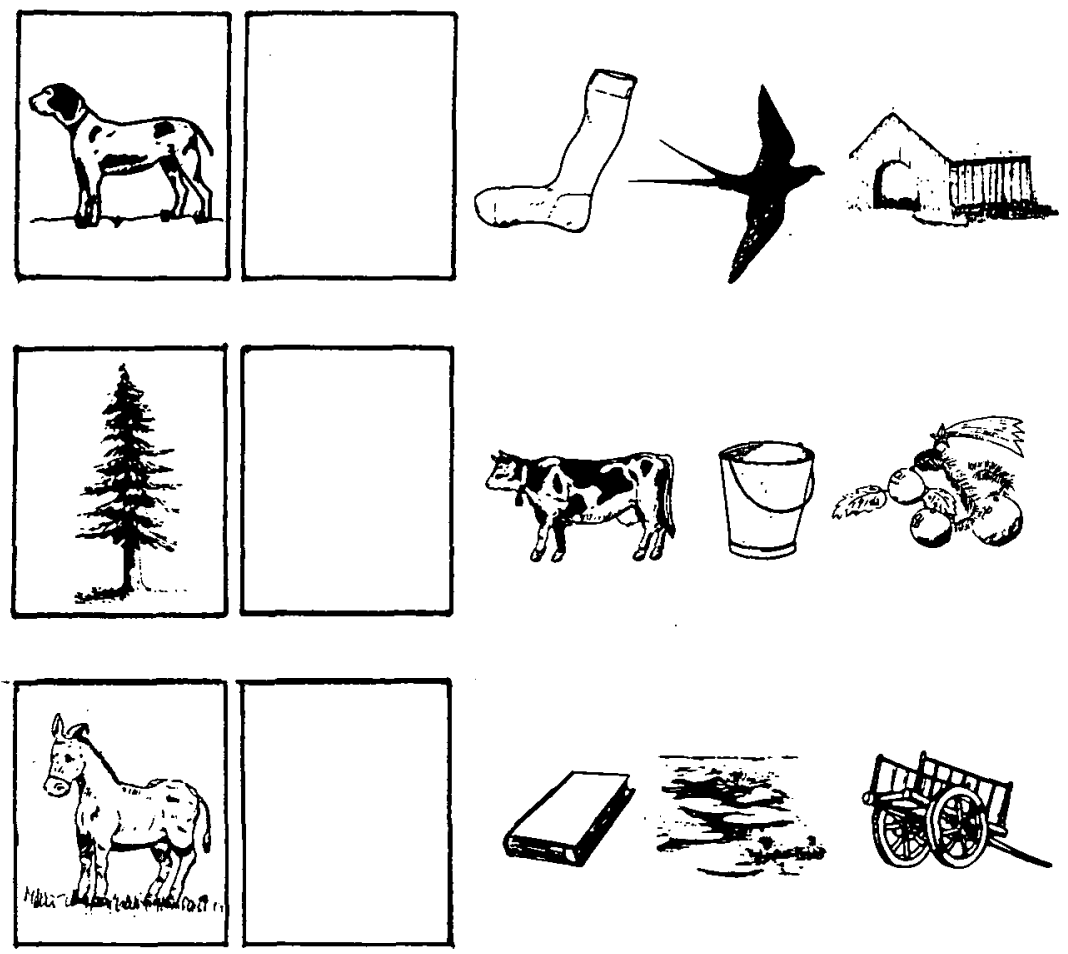

Ejemplos de láminas de emparejamiento. Aparecen las tres posibles agrupaciones categoriales: animal-animal; planta-planta; $y$ animal-planta. En cada una de ellas existía además la posibilidad de emparejamiento contextual: perro-caseta, árbol-adornos y burro-carro.

ayudaba en la tarea. La fase de recuerdo se realizó el día siguiente a la presentación del material y en ella se pedía a los sujetos que recordasen el mayor número posible de los dibujos que habian visto el día anterior.

En la prueba de emparejamiento se presentaron 13 láminas con la estructura descrita con anterioridad. De éstas, las tres primeras se utilizaron como láminas de prueba, con la intención de que el sujeto comprendiese la tarea, a la vez que se inducía la elección categorial. De hecho, durante la fase de prueba no se pasaba a la lámina siguiente hasta que el sujeto no hubiera realizado la elección categorial y la hubiese explicado sobre la base del concepto de vida.

\section{Indices de medida}

Las fases de estudio de las pruebas de memoria se codificaron de acuerdo con dos sistemas de categorías. Uno de ellos hacía referencia a las conductas verbales y otro a las acciones de estudio.

\section{Acciones de estudio}

1. Examen del material. El sujeto mira el material sin modificar su organización, independientemente de que lo manipule o no. Dentro de esta 
Figura 2


Ejemplos del material utilizado en las pruebas de memoria. Al igual que en la prueba de emparejamiento los estimulos son susceptibles de ser agrupados bajo criterio contextual (cigüeña-nido; olla-cuchillo-lechuga, etc.) o categorial (cigüeña-toro; casa-nido; etc.).

categoría se consideran conductas como contar el material, inspección visual, señalar y/o coger las tarjetas (o cualquier otra forma de manipulación).

2. Repaso o autoexamen. El sujeto deja de mirar el material durante un intervalo de tiempo, volviendo inmediatamente después a mirarlo.

3. Agrupación. El sujeto modifica la disposición de las tarejas formando uno o varios grupos de dos o más elementos.

4. Apoyo social. El sujeto dirige su mirada al investigador.

5. Conductas de no estudio Se incluyen en este apartado las conductas relacionadas con interrupciones de la prueba o distracciones del sujeto. 


\section{Conductas verbales}

Las categorías empleadas para analizar el componente verbal de las acciones se describen en el artículo de Ramírez et al. (en este mismo volumen), por lo que remitimos a él.

Para el análisis del recuerdo se utilizaron diversos índices de medida:

a) Número total de ítems recordados correctamente (NIR).

b) Ajusted Ratio of Clustering (ARC) como medida de la agrupación en el recuerdo (Roenker, Thompson y Brown, 1971).

c) Diference Score (DS), como medida global que combina la cantidad de recuerdo con el grado de agrupación del mismo (Bousfield y Bousfield, 1966).

\section{RESULTADOS}

\section{Pruebas de emparejamiento}

Los análisis realizados mostraron una influencia estadísticamente significativa de la escolarización en las destrezas de categorización. Los sujetos del nivel de alfabetización, los de menor experiencia escolar, obtuvieron resultados inferiores a los de los niveles educativos superiores, pregraduado y graduado $(F=3,074 ; p=0,0289)$. El test de rango múltiple de Scheffé para $\alpha=0,05$ mostró al nivel de alfabetización como generador de las diferencias (Tabla I, Figura 3).

\section{TABLA I}

Puntuaciones medias de los niveles educativos en las pruebas de emparejamiento $y$ memoria

\begin{tabular}{|c|c|c|c|c|c|c|}
\hline \multirow{2}{*}{$\begin{array}{l}\begin{array}{l}\text { Nivel } \\
\text { Educativo }\end{array} \\
\text { Fase }\end{array}$} & \multicolumn{2}{|c|}{ Alfabetización } & \multicolumn{2}{|c|}{ Pregraduado } & \multicolumn{2}{|c|}{ Graduado } \\
\hline & M-1 & M-2 & M-1 & M-2 & M-1 & M-2 \\
\hline $\begin{array}{l}\text { Emparejamiento } \\
\text { Indice de memoria }\end{array}$ & & & & & & \\
\hline $\begin{array}{l}\text { D.S. } \\
\text { A.R.C. } \\
\text { N.I.R. }\end{array}$ & $\begin{array}{r}1,90 \\
0,39 \\
12,04\end{array}$ & $\begin{array}{l}1,85 \\
0,18 \\
9,68\end{array}$ & $\begin{array}{r}3,50 \\
0,44 \\
14,79\end{array}$ & $\begin{array}{r}4,40 \\
0,52 \\
14,75\end{array}$ & $\begin{array}{r}5,64 \\
0,65 \\
16,25\end{array}$ & $\begin{array}{r}5,27 \\
0,73 \\
14,00\end{array}$ \\
\hline
\end{tabular}

\section{La Prueba de memoria}

\section{Fase de Presentación}

Como hemos señalado con anterioridad, aunque el análisis de esta fase contemplara la codificación de conductas tanto verbales como no verbales, en este artículo no vamos a considerar las primeras (un análisis detallado de éstas se puede encontrar en el artículo de Ramírez et al., que aparece en este mismo número). 
FIGURA 3

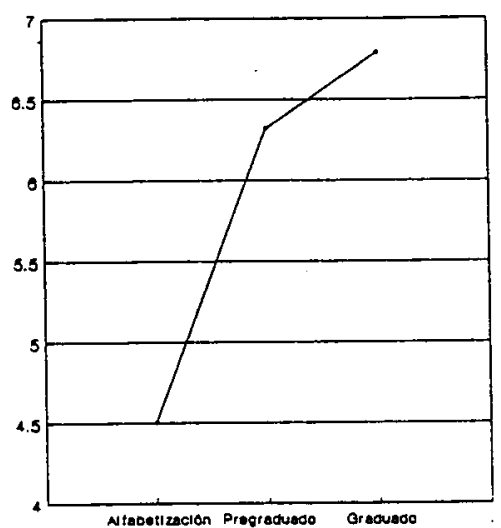

Prueba de emparejamiento.

Los datos obtenidos mostraron una base común en el modo de enfrentarse al estudio del material. Como era lógico suponer, la categoría de examen destacó sobre las demás, a la vez que no se apreciaron diferencias significativas entre los grupos (Tabla II, Figura 4.1). Las diferencias aparecieron al considerar las acciones de marcado carácter estratégico, el repaso y la agrupación. En la primera de ellas, las diferencias significativas las estableció el grupo de alfabetización, con resultados significativamente inferiores a los obtenidos por los de pregraduado $(\mathrm{z}=3,993, \mathrm{p}<0,01)$ y graduado $(\mathrm{z}=2,639, \mathrm{p}<0,01)$. En cambio, en el uso de la agrupación fue el grupo de graduado el que estableció las diferencias respecto de pregraduado $(\mathrm{z}=3,432, \mathrm{p}<0,01)$ y alfabetización $(\mathrm{z}=1,888, \mathrm{p}<0,05)$, logrando puntuaciones significativamente superiores.

FIGURA 4.1

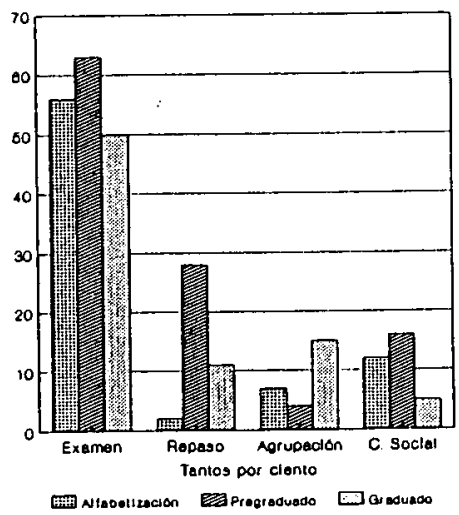

Acciones de Estudio.

Primer periodo de estudio.
FIGURA 4.2

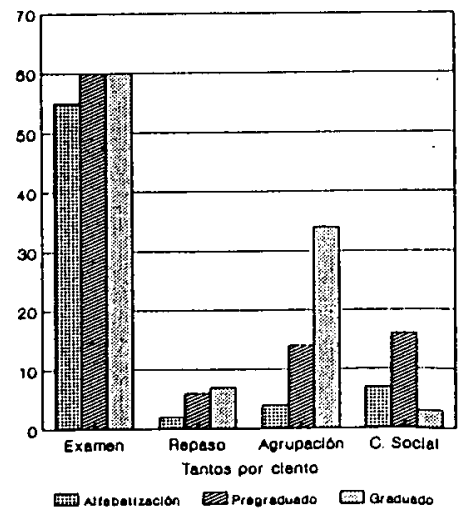

Acciones de Estudio. Segundo periodo de estudio. 
La categoría de apoyo social no estableció diferencias significativas entre los grupos en cuanto al número de veces que se hizo uso de la misma. Sin embargo, el tipo de conductas que se engloban bajo esta categoría es cualitativamente distinto en función del nivel de escolarización de los sujetos. Aunque una discusión más detallada de este aspecto se presenta en el artículo de Ramírez et al. (en este volumen), es interesante comentar que mientras que los sujetos de graduado hacían preguntas sobre las condiciones de resolución de la tarea, en los niveles inferiores predominaban miradas en busca de aprobación o que denotaban falta de dominio de la situación y la tarea.

TABLA II

Número de intervalos en los que se usaron las distintas acciones de memoria

\begin{tabular}{l|rr|rr|rr}
\hline $\begin{array}{l}\text { Nivel } \\
\text { Educativo }\end{array}$ & \multicolumn{2}{|c|}{ Alfabetización } & \multicolumn{2}{|c|}{ Pregraduado } & \multicolumn{2}{c}{ Graduado } \\
\hline Fase & M-1 & M-2 & M-1 & M-2 & M-1 & M-2 \\
\hline Acción de memoria & & & & & & \\
Examen & 56 & 55 & 63 & 60 & 50 & 60 \\
Repaso & 2 & 2 & 28 & 6 & 11 & 7 \\
Agrupación & 7 & 4 & 4 & 14 & 15 & 34 \\
C. Social & 12 & 7 & 16 & 16 & 5 & 3 \\
\hline
\end{tabular}

Fase de recuerdo

Los análisis del recuerdo también mostraron diferencias significativas en función del nivel de escolarización, si bien dependiendo del índice de recuerdo utilizado, las diferencias fueron establecidas por uno u otro grupo (Tabla I). El análisis factorial univariable de las puntuaciones DS (índice mixto de carácter global) mostró una diferencia significativa en función de escolarización ( $F=17,7396 ; p=0,00001)$. El test de rango múltiple de Scheffé indicó un escalonamiento en las puntuaciones, es decir, las puntuaciones significativamente más bajas $(\alpha=0,05)$ las obtuvo el grupo de alfabetización, seguido de pregraduado y significativamente por encima de éste el grupo de graduado (Figura 5.1). Este escalonamiento, sin embargo, no se mantiene en los resultados de los otros dos índices de recuerdo empleados, NIR y ARC. Si bien las puntuaciones en ambos variaron significativamente en función del nivel de escolarización (NIR: $F=8,6024$; $\mathrm{p}=0,0004$; ARC: $\mathrm{F}=7,8422, \mathrm{p}=0,0008$ ), el grupo que marcó las diferencias fue distinto según se utilizara un índice u otro. En el caso del NIR, es decir cuando nos centramos en los aspectos puramente cuantitativos del recuerdo, las diferencias las aportó el grupo de alfabetización, con puntuaciones significativamente inferiores a las de los dos niveles restantes, entre los cuales no se hallaron diferencias significativas (siempre utilizando el test de rango múltiple de Scheffé para $\alpha=0,05$ ) (Figura 5.2). La tendencia es la inversa al analizar los aspectos cualitativos del recuerdo; las diferencias significativas en el índice ARC las estableció el grupo de graduado con puntuaciones superiores a las de pregraduado y alfabetización, entre las cuáles no se encontraron diferencias (Figura 5.3). 
FIGURA 5.1

- Fase I T Fase II



Prueba de memoria.

Indice: Diference Score.
FIGURA 5.2

- Fase I T Fase II

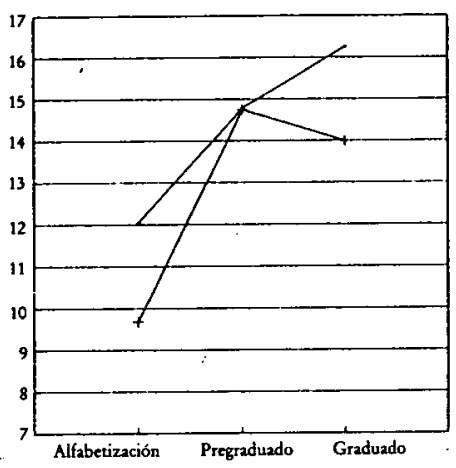

Prueba de memoria.

Indice: N. ${ }^{\circ}$ de Items Recordados.

\section{Segunda Prueba de memoria}

\section{Fase de Presentación}

Los análisis de la segunda fase de presentación mostraron cambios significativos en las categorías de repaso y agrupación, es decir, aquéllas que englobaban las acciones de carácter más marcadamente estratégico. Tanto en pregraduado como en graduado se apreció un aumento significativo en la categoría de agrupación $(z=2,625, p<0,01$ y $z=1,694, p<0,01$ respectivamente). La categoría de repaso, por el contrario, mostró una disminución significativa en su frecuencia de aparición $(z=3,483, p<0,01$ y $\mathrm{z}=1,696, \mathrm{p}<0,05)$ en ambos grupos. Por su parte, el grupo de alfabetización no presentó diferencias significativas en ninguna de las categorías de

\section{FIGURA 5.3}

- Fase $1+$ Fase II

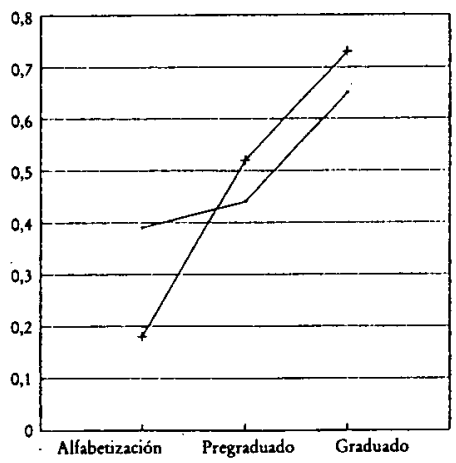

Prueba de memoria.

Indice: Adjusted Ratio of Clustering. 
análisis. De este modo, las diferencias encontradas en la primera fase en la categoría de repaso desaparecieron en la segunda, mostrando todos los niveles índices similares. En la categoría de agrupación se produjo un escalonamiento en los resultados, de tal modo que aparecieron diferencias significativas entre los niveles de alfabetización y pregraduado $(z=1,967$, $\mathrm{p}<0,05$ ), inexistentes en la primera prueba. El grupo de graduado continuó presentando los mayores índices de agrupación, obteniendo puntuaciones significativamente más elevadas que las de los grupos de alfabetización $(\mathrm{z}=4,199, \mathrm{p}<0,01)$ y pregraduado $(\mathrm{z}=2,752, \mathrm{p}<0,01)$ (Tabla II, Figura 4.2).

\section{Fase de recuerdo}

En esta segunda fase de recuerdo no se encontraron avances significativos respecto del recuerdo de la primera lista ${ }^{2}$. Sin embargo, parece que la prueba de emparejamiento ejerció influencias diferenciales en los diversos grupos. Al igual que en la primera fase, se hallaron diferencias significativas con todos los índices de recuerdo empleados (DS: $F=8,8213$; $p=0,0003 ;$ NIR: $F=7,769 ; p=0,00008 ;$ ARC: $F=12,0726$, $\mathrm{p}=0,00001)$. El test de rango múltiple de Scheffé para $\alpha=0,05$ mostró importantes cambios respecto de la fase anterior. Con DS como índice, las diferencias entre los grupos de pregraduado y graduado desaparecieron, manteniéndose las de ambos grupos respecto del nivel de alfabetización (Figura 5.1). Utilizando el NIR como índice, no hubo cambios en relación con la primera fase, de tal modo que se encontraron diferencias significativas entre el nivel de alfabetización y los dos niveles restantes, pregraduado y graduado. Entre éstos no se hallaron diferencias significativas (Figura 5.2). El índice ARC, en cambio, también mostró diferencias respecto de la primera prueba de memoria. Si en la primera fase las diferencias las establecía el grupo de graduado respecto de las anteriores, en ésta se encontraron unos resultados escalonados, de tal modo que se apreciaron diferencias significativas entre los niveles de alfabetización y pregraduado (que no aparecian en la primera prueba), y entre éste último y el grupo de graduado (Figura 5.3).

\section{DISCUSION Y CONCLUSIONES}

En general, podemos anticipar que los resultados avalan las hipótesis enunciadas previamente. La experiencia en situaciones formales como la escolarización promueve el empleo de destrezas, acciones, de memoria de carácter estratégico y descontextualizado. Esto se pone de manifiesto en los dos tipos de análisis apuntados: el relativo a las diferencias entre los grupos de escolarización y el efectuado en relación al componente microgenético del trabajo. La discusión irá dirigida a sustentar estas afirmaciones.

\section{Efectos de la escolarización sobre la agrupación y la memoria}

Los resultados de la prueba de emparejamiento nos muestran un mayor empleo de la agrupación categorial en los grupos de pregraduado y graduado. Ambos alcanzan niveles muy semejantes, por encima del alcanzado por 
los sujetos de alfabetización. Podemos afirmar que los sujetos de ambos grupos demuestran un dominio similar de la agrupación categorial cuando ésta es el objetivo de la tarea, es decir, cuando la agrupación se sitúa en el plano de las acciones. Frente a ellos, los sujetos de alfabetización dominan en menor medida estas acciones. A pesar de que se les inducía a agrupar los elementos sobre la base del concepto de vida, cuando el material exigía trabajar en los niveles más descontextualizados de dicho concepto (específicamente, emparejar dibujos de animales y plantas), estos sujetos no mantenían el objetivo de agrupación por el criterio «ser vivo".

En relación a los resultados de memoria, se observa que el nivel escolar va asociado al empleo progresivo de destrezas de índole estratégica. Esto se pone de manifiesto al interpretar las diferencias entre el nivel de alfabetización y los de pregraduado y graduado. Igualmente, los resultados muestran que los sujetos del nivel de graduado emplearon acciones más complejas que los de pregraduado y alfabetización. Estos resultados coinciden con la mayoría de las investigaciones que han estudiado la relación entre escolarización y memoria (Cole et al., 1971; Cole y Scribner, 1974; Rogoff, 1981; Rogoff y Mistry, 1985; Sharp, Cole y Lave, 1979; Wagner, 1981). Podemos afirmar, en la línea de los trabajos de Paris y colaboradores, que el empleo de acciones de memoria está relacionado con una variable macrosocial como la escolarización.

Avanzando en la interpretación de los resultados, podría afirmarse que el uso de acciones estratégicas complejas conlleva un desligamiento por parte del sujeto respecto del material y la tarea. Si analizamos la situación de memorización de nuestra prueba, encontramos que, enfrentado al problema que se le plantea, el sujeto puede emplear procedimientos que modifiquen en mayor o menor medida los elementos dados en dicha situación. Cuando los sujetos de alfabetización típicamente repiten en voz alta el nombre de los dibujos sin alterar la disposición de las tarjetas sobre la mesa, están apartándose muy poco de la situación que se les propone. Incluso el nombrar los dibujos en voz alta parece responder a un seguimiento directo de las instrucciones dadas por el experimentador, con muy poca evidencia de que estos sujetos asuman realmente el objetivo de memorizar propuesto ${ }^{3}$. Podemos decir, por tanto, que los sujetos de alfabetización demuestran poco desligamiento de la situación. Esta falta de desligamiento se pone de manifiesto de dos maneras: existe un alto grado de heterorregulación en sus acciones (lo que se trata con mayor profundidad en el trabajo de Ramírez et al.); y esta heterorregulación va acompañada de una dependencia marcada del material, con escasa modificación de los elementos dados. La agrupación, por su parte, indica un desligamiento de la situación, en los dos sentidos apuntados: autorregulación e imposición de una nueva estructura al material, patente incluso cuando la agrupación tiene un carácter asociativo. Si bien en este punto hemos utilizado el término desligamiento, una interpretación más profunda de estos hallazgos nos remitiría a la noción de descontextualización. Hemos reservado este término para darle un carácter plenamente sociocultural. Volveremos sobre esta cuestión más adelante.

\section{Análisis microgenético}

De modo general, se aprecian dos patrones de actuación distintos en la realización de las tareas. El primero de ellos no presenta cambios cualita- 
tivos, manteniéndose relativamente estable a lo largo de los cuatro días que comprendía el estudio. Corresponde tanto a los niveles de graduado como de alfabetización. La estabilidad observada en ambos responde, sin embargo, a modos distintos de enfrentarse a la tarea. En el grupo de graduado, el dominio de las acciones de agrupación está bastante desarrollado. En el grupo de alfabetización, por su parte, el dominio de estas acciones es prácticamente nulo. Como se señaló anteriormente, la mayoría de las elecciones realizadas en la prueba de emparejamiento estuvieron basadas en un criterio contextual. La ausencia de agrupación categorial taxonómica dominó igualmente en la prueba de memoria, como muestran las bajas puntuaciones obtenidas por este último grupo cuando se utilizó el ARC como índice de recuerdo.

El segundo patrón hace referencia a la actuación de los sujetos del nivel de pregraduado. Lo que caracteriza a éste frente al anterior son los cambios sustanciales que se producen a lo largo del estudio en el empleo de acciones de agrupación. Detengámonos a analizar su actuación.

Como se ha indicado anteriormente, la prueba de emparejamiento mostró a unos sujetos bastante diestros en el empleo de acciones de categorización cuando el objetivo de la tarea era precisamente el de categorizar $(63,2 \%$ de elecciones categoriales frente al $67,8 \%$ de graduado y al $43,9 \%$ de alfabetización). El hecho de que no se diferenciasen significativamente de los sujetos con una alta experiencia escolar, y si lo hicieran de los que poseen escasa o nula, hace pensar que la escolarización deja sentir su influencia en el dominio de tales acciones tempranamente. Diferente es el caso del empleo de acciones de agrupación al servicio de la memoria, lo que implica su subordinación a objetivos más generales. En la primera fase, los alumnos de pregraduado mostraron en pocas ocasiones conductas de agrupación durante la fase de estudio $y$, consecuentemente obtuvieron unos índices de agrupación muy bajos en la fase de recuerdo de esta primera prueba. Tras la realización de la prueba de emparejamiento, los resultados obtenidos fueron especialmente relevantes, obteniéndose un aumento significativo en el empleo de la acción de agrupación durante la fase de estudio de la segunda prueba de memoria. El análisis más pormenorizado de la actuación en el recuerdo nos permite entender con más claridad los procesos implicados. Centrándonos en el número de elementos recordados, el patrón de resultados muestra una superioridad de los niveles de pregraduado y graduado, sobre el de alfabetización, sin que existieran diferencias entre los primeros. Esta diferencia se mantiene en la segunda fase. Lo observado al contemplar el patrón de agrupación en sí mismo (ARC) es, sin embargo, distinto. En la fase de recuerdo I no aparece una diferencia significativa entre los niveles de agrupación en el recuerdo que muestran los sujetos de pregraduado y los de alfabetización, siendo inferiores ambos a los de graduado. En la fase de recuerdo II, en cambio, los resultados de pregraduado son significativamente superiores a los de alfabetización, aunque no llegan a igualarse a los de graduado. Estos datos nos hacen pensar que el aprendizaje de acciones de memorización voluntaria de información discreta incluye diferentes aspectos. El manejo intencional de información discreta en ss aspectos más cuantitativos parece estar suficientemente establecido tras un período no excesivamente largo de experiencia escolar. Más complejos y por tanto, de adquisición más tardía en el curso de la escolarización, pa- 
recen ser los aspectos que implican el manejo de criterios categoriales en el recuerdo voluntario. La desaparición de las diferencias entre los sujetos de pregraduado, y los de alfabetización y graduado, motivada por la mejora de los primeros tras la fase de emparejamiento, da idea de un proceso de adquisición gradual de estas acciones en el curso de la escolarización. Este dato es coherente con el predominio del uso de acciones de repaso entre los sujetos de pregraduado durante la primera fase de estudio y el mayor empleo de acciones de agrupación por parte de estos mismos sujetos durante la segunda fase. Mientras que el uso de acciones de repaso puede ser suficiente para recordar un elevado número de elementos, la agrupación en el recuerdo parece reflejar el empleo de acciones de agrupación durante las fases de estudio. En otro dominio genético, como es la ontogénesis, los resultados obtenidos por diferentes autores indican que las acciones de repaso se adquieren en edades más tempranas que las de agrupación (Kail, 1979).

\section{CONCLUSIONES}

La subordinación de las acciones de agrupación al servicio de la memorización y el recuerdo, que se pone de manifiesto al analizar los datos microgenéticos, es uno de los resultados más interesantes de este trabajo. Podemos interpretarlos como un ejemplo de cambio en la organización dinámica de las funciones mentales (Wertsch, 1985a). Si consideramos a la acción como la unidad de análisis de la conciencia, los cambios en las relaciones entre las acciones conllevan cambios cualitativos en la organización de la conciencia. La agrupación se convierte en una acción guiada por un nuevo objetivo: la memoria. Se agrupa para memorizar y recordar y este objetivo de memoria está presente al realizar la agrupación. La memorización y el recuerdo, por su parte, implican agrupación. Cuando el sujeto utiliza el criterio categorial para recordar los elementos, esta acción de recuerdo no es únicamente una reinstauración de los materiales presentados previamente, es también una reconstrucción de los recuerdos utilizando las relaciones categoriales entre los elementos. Por tanto, el empleo de la agrupación categorial como acción subordinada a la memoria supone no sólo un cambio en la relación existente entre ambas, sino también una modificación de estas funciones mismas. En el caso de nuestro estudio, pues, el análisis microgenético del grupo de pregraduado nos permite observar cómo cambia una función mental superior en este contexto: unas funciones mentales superiores rudimentarias evolucionan hacia estadios avanzados (Vygotsky, 1979; Wertsch, 1985a). El criterio que permite diferenciar las funciones mentales superiores rudimentarias avanzadas en el empleo de procedimientos de mediación descontextualizados. Este cambio en los procedimientos semióticos de mediación debe estar en la base de los desarrollos observados. ¿En qué sentido podemos afirmar que en nuestro trabajo se pone de manifiesto la intervención de procedimientos descontextualizados de mediación semiótica de las acciones? El empleo de criterios categoriales como «ser vivo», «animal», o «vivienda» implica funcionar, no sobre las relaciones que se dan entre los objetos en la vida cotidiana, sino sobre los significados verbales. El significado verbal de «ser vivo" incluye otros significados verbales como "animal», "planta», «mamífero" o "perro». In- 
dependientemente de que los objetos reales designados con estas palabras mantengan relaciones en la vida cotidiana, los significados verbales citados sí las poseen, de modo que un significado verbal descontextualizado como los anteriores remite a los demás. Es en este sentido en el que afirmamos que la agrupación categorial taxonómica implica unos procedimientos descontextualizados de mediación. La utilización de la agrupación categorial como instrumento de memoria implica, además, la elaboración y empleo de un plan de acción, que sólo es posible sobre esta base. El uso de significados verbales descontextualizados permite esta nueva relación entre agrupación y memoria. Pero no sólo cambia la relación, también cambian la agrupación y la memoria mismas. La resolución categorial de la prueba de emparejamiento implica el empleo de significados verbales descontextualizados como instrumento mediador de la acción. Subordinar la agrupación a la memoria implica también un plan de acción en el que la agrupación se convierte en instrumento para la memorización y el recuerdo. Probablemente no sea posible esta reorganización funcional sin la intervención del potencial descontextualizador del lenguaje. El uso de significados verbales descontextualizados permite «objetivar» las acciones, haciéndola menos dependiente del material y del conjunto de la situación. Esto facilita el manejo de la acción como un instrumento útil en diferentes contextos y al servicio de distintos objetivos. Esta percepción de la acción como algo separado de la situación en que se emplea, que permite su observación por parte del sujeto, se relaciona con lo que se denomina metacognición, vincula$\mathrm{da}$, como muestran muchos estudios, con las experiencias educativas formales.

\section{Apuntes para futuras investigaciones}

La investigación que aquí hemos desarrollado es un intento de establecer algunas diferencias ligadas a la experiencia escolar en la adquisición y empleo de acciones de agrupación y memoria. A pesar de que los análisis microgenéticos son coincidentes con los resultados de la comparación por niveles educativos, es evidente que las diferencias entre dichos niveles pueden deberse a muchos factores de experiencia cultural, aparte de la escolarización. Esta cuestión, que ha preocupado a muchos autores no es, sin embargo, lo que más nos preocupa. Si consideramos a los grupos de escolarización como representantes de distintos tipos de experiencia en actividades socioculturales, con mayor incidencia de actividades formales y modernas en el grupo de graduado y predominio de experiencias en actividades tradicionales e informales en el de alfabetización, la cuestión queda planteada en otros términos. Haciendo esta interpretación sociocultural, lo importante es estudiar en qué medida la participación en actividades modernas o «racionalizadas» (Weber, 1978; Wertsch, 1985a) modifica las funciones mentales. Planteada la cuestión de ese modo, creemos que nuestro trabajo resulta de interés.

No obstante lo dicho, el conocimiento de la influencia de la educación formal en los procesos de pensamiento requiere ir más allá de los estudios de caja negra (Mehan, 1979) predominantes hasta la fecha. Conocemos con cierta precisión las características del pensamiento de los individuos socializados en contextos informales, y conocemos en cierta medida en qué sen- 
tido ejerce su influencia la experiencia educativa formal en el mismo. Sin embargo, sabemos muy poco acerca de los mecanismos por los que un proceso cultural como la escolarización deja sentir su influencia en los procesos psicológicos individuales. Intentar cubrir esta laguna requiere, no cabe duda, de un importante esfuerzo tanto teórico como metodológico.

El estudio del discurso en las interacciones educativas es, a nuestro entender, la vía que puede permitirnos desvelar la naturaleza de los mecanismos semióticos e interactivos que están en la base de los cambios cognitivos generados por actividades socioculturales como la educación formal. Nuestros trabajos actuales se desarollan en esta línea ${ }^{4}{ }^{5}$.

\section{Notas}

1 En el momento de la recogida de datos estos eran los niveles educativos más comunes a todos los centros, si bien en algunos de ellos existían otros niveles tales como postalfabetización, neolectores, etc. Los contenidos curriculares de estos niveles podrían coincidir con los de los ciclos de EGB: alfabetización-ciclo inicial; pregraduado-ciclo medio; y graduado-ciclo superior.

${ }_{2}$ Esta ausencia de avance en la segunda fase puede ser debida al gran número de dibujos que habían sido presentados hasta el momento a los sujetos, siendo todos ellos de características muy similares. Este hecho produjo un gran número de invenciones que generaron el lógico descenso en las puntuaciones de la segunda fase.

3 Todas estas conductas verbales tienen una importante significación psicológica. Se tratan con más detenimiento en el artículo de Ramírez et al., en este mismo número.

4 Ramírez, J. D. y L. A. H.: Lenguaje y pensamiento en situaciones de cambio cultural: una aproximación etnometodológica al análisis del discurso en la educación de adultos. Proyecto subvencionado por la DGICYT, 1988.

${ }^{5}$ Ramírez, J. D., L. A. H. y otros: La educación de adultos y el cambio de valores, actitudes y modos de pensamiento en la mujer: un estudio etnográfico del discurso. Proyecto subvencionado por el CIDE, 1989.

\section{Referencias}

BOUSFIELD, A. K. y BOUSFIELD, W. A. (1966). Measurement of clustering and of sequential constancies in repeated free recall. Psychological Reports, 19, 935-942.

BROWN, A. L. (1977). Development, schooling and the acquisition of knowledge about knowledge. En R. C. Anderson, R. J. Spiro y W. E. Montague (eds.) Schooling and the acquisition of knowledge. Hillsdale, NJ: LEA.

COLE, M. (1984). La zona de desarrollo próximo: donde cultura y conocimiento se generan mutuamente. Infancia y Aprendizaje, 25, 3-17.

COLE, M., GAY, J., GLICK, J. y SHARP, D. W. (1971). The cultural context of learning and thinking. Nueva York: Basic Books.

COLE, M. y SCRIBNER, S. (1974). Culture and thought: A psychological introduction. Nueva York: Wiley (Trad. castell. en México: Limusa, 1977).

FlavelL, J. H. (1970). Developmental studies of mediated memory. En H. W. Reese y Lipsitt (eds.) Advances in child development and behavior. Vol. III. Nueva York: Academic Press.

GAUVAIN, M. y RogOFF, B. (1987). Ways of speaking about space: the development of children's skills at communicating spatial knowledge. Trabajo presentado en el Biennial Meeting of the Society for Research in Child Development, Baltimore.

GREENFIELD, P. M. (1984). A theory of the teacher in the learning activities of everyday life. En B. Rogoff y J. Lave (eds.) Everyday Cognition. Its development in Social Contexts. Cambridge, Mass.: Harvard University Press.

GrEeNFIELD, P. M. y LAVE, J. (1982). Cognitive aspects of informal education. En D. Wagner y H. Stevenson (eds.): Cultural Perspectives on Child Development. San Francisco. Freeman.

KalL, R. V. (1979). The development of memory in children. San Francisco: Freeman \& Co.

LABORATORY OF COMPARATIVE HUMAN COGNITION (LCHC) (1983). Culture and cognitive development. En W. Hessen (ed.) Handbook of child psychology. Vol. I. Nueva York: Wiley. 
Leontiev, A. N. (1973-74). The problem of activity in psychology. Soviet Psychology, 13, 4-33.

Leontiev, A. N. (1981). Problems of the development of the mind. Moscu: Progress Publisher.

LURIA, A. R. (1979). Los procesos cognitivos. Análisis sociobistórico. Barcelona: Fontanella.

MANDlER, J. M., SCRIBNER, S., COLE, M. y DeForeST, M. (1980). Cross-cultural invariance of story recall. Child Development, 51, 19-26.

De la MATA, M. L. (1988). El desarrollo de las acciones de memoria. Infancia y Aprendizaje, 42, 3-18.

De LA MATA, M. L. y RAMirez, J. D. (1989). Cultura y procesos cognitivos: hacia una psicología cultural. Infancia y Aprendizaje, 46, 49-70.

MeAHN, H. (1979). Learning lessons. Social interaction in the classroom. Cambridge, Mass.: Harvard University Press.

PARIS, S. G., LIPSON, M .Y. y WiKXON, K. K. (1983). Becoming a strategic reader. Contemporary educational psychology, 8, 293-316.

PARIS, S. G., NEWMAN, R. S. y JACOBS, J. E. (1985). The social context and functional of children's remembering. En M. Pressley y C. J. Brainerd (eds.) Cognitive learning and memory in children. Nueva York: Springer-Verlang.

RoENKER, D. L., THOMPSON, C. P. y BROWN, S. C. (1971). Comparisons of measures for the estimation of clustering in free recall. Psychological Bulletin, 76, 45-48.

ROGOFF, B. (1981). Schooling and the development of cognitive skills. En H. C. Triandis y A. Heron (eds.) Handbook cross-cultural psychology. (Vol. IV). Boston: Allyn \& Bacon.

ROGOFF, B. (1982). Integrating context and cognitive development. En M. E. Lamb y B. Rogoff (eds.) Advances in developmental psychology. Vol. II. Hillsdale, NJ: LEA.

ROGOFF, B. y GARDNER, W. (1984). Adult guidance of cognitive development. En B. Rogoff y J. Lave: Everyday cognition. Its development in social contexts. Cambridge, Mass.: Harvard University Press.

RoGOFF, B. y MiSTRY, J. (1985). Memory development in cultural context. En M. Pressley y C. J. Brainerd (eds.) Cognitive learning and memory in children. Nueva York: Springer-Verlang.

SCRIBNER, S. (1974). Developmental aspects of categorized recall in West Africa. Cognitive Psychology, 6, 475-494.

SCRIBNER, S. y COLE, M. (1981). The psychology of literacy. Harvard University Press.

SCRIBNER, S. y COLE, M. (1973). Cognitive consequences of formal and informal education. Science, 182, 553-559 (Trad. castell. en Infancia y Aprendiżaje, 1982, 17, 3-18).

ShaRP, D., COLE, M. y LAVE, C. (1979). "Education and cognitive development: The evidence from experimental research." Monographs of the Society for Research in Child Development, 44 (1-2 Serial $\left.n .^{\circ} 178\right)$.

TRIANDIS, H. C. y HERON, A. (1981). (Eds.) Handbook of cross-cultural psychology. (Vol. IV). Boston: Allyn \& Bacon.

Vygotski, L. S. (1973). Pensamiento y Lenguaje. Buenos Aires: La Pléyade.

Vygotski, L. S. (1979). El Desarrollo de los Procesos Psicológicos Superiores. Barcelona: Crítica.

WAGNER, D. A. (1981). Culture and memory development. In H. C. Triandis.y A. Heron (eds.) Handbook Crosscultural Psychology. (Vol. IV). Boston: Allyn \& Bacon.

WERTSCH, J. V. (1981). The concept of activity in Soviet Psychology. White Plains: Sharpe.

WERTSCH, J. V. (1985a). Vygotsky and the social formation of the mind. Nueva York: Harvard University Press.

WeRTSCH, J. V. (1985b). The semiotic mediation of mental life; L. S. Vygotsky and M. M. Bakhtin. En E. Mertz y R. J. Parmentier. Semiotic mediation. Sociocultural and psychological perspectives. Nueva York: Academic Press.

WERTSCH, J. V., MINICK, N. y ARNS, F. J. (1984). The creation of context in joint problem solving. En B. Rogoff y J. Lave (eds.) Everyday Cognition. Its development in social context. Cambridge, Mass.: Harvard University Press.

ZIVIAN, M. T. y DARJES, R. W. (1983). Free-recall by in-school and out-of-school adults: Performance and metamemory. Developmental Psychology, 22, 2, 513-520. 


\section{Extended Summary}

The purpose of this work is to study the influence of schooling on the development of cognitive processes (e.g., categorization and memory), as well as on the verbal regulation of those processes.

This work is based on a socio-cultural approach and takes the Soviet concept of action as the unit of analysis. Namely, we study the influence of school experience on the acquisition and use of categorization and memory actions.

Our conceptualization of memory actions is related to Paris'. Paris uses the term strategy as synonimous of action and emphasizes its contextualized and socialized nature.

This work adopts a double cross-sectional and microgenetic perspective. On the one hand, we selected subjects from three different educational levels taking an adult education regional program; on the other hand, our methodology consists of three successive phases. The first and third phases involved presentation and free recall of two sets of categorizable items. The intermediate phase was a directed categorization task, in which similar material was used. This three-phase methodology allows us to observe the development of clustering skills throughout these phases. In the two recall tasks, clustering skills should work as a means for memorizing and recalling the stimuli. That is, the clustering skills were subordinate actions (i.e., included into a more general memory action). In turn, clustering skills work as independent actions in the second phase, because they were not subordinate to any other goal. We expected that the training in the directed categorization task would facilitate the use of clustering skills, so that subjects who were not able to employ these skills to remember in the first recall task could use them in the third phase. We expected that practice in using clustering as an independent action would increase subjects' mastery of these actions and, therefore, make possible their subordination into a more general (memory) goal.

Selection of adult subjects was based on several theoretical reasons. The use of adult subjects provides us with the opportunity to study the influence of a cultural experience like formal education (i.e., a cultural line of development) when biological development (i.e., the natural line) has been completed.

The study of memory and clustering actions, from a Vygotskian sociocultural approach, underscores the necessity of taking into account the mediated character of actions. In our work, we analyze verbalizations occurring during the task. In order to study speech in the course of actions, we employed a double analysis of subjects' performance during study periods in the first and the third phases. This double analysis included memory actions and speech regulating them.

\section{METHOD}

\section{Subjects}

The study sample were 105 subjects from different adult education schools in the city of Seville. They were recruited from three educational levels: Illi- 
terate, pregraduate and elementary school graduates. Most of them $(85.7 \%)$ were women between 45 and 60 years of age; only $14.3 \%$ were men, reflecting approximate sex proportions in adult education.

\section{Procedure}

The methodology consisted of three successives phases:

1) Presentation and recall of categorizable stimuli $A$. Subjects were presented with a set of cards depicting 22 items that were likely to be categorized on the basis of taxonomic as well as associative or functional criteria. The study period was videotaped. On the second day, subjects were asked to recall the pictures.

2) Directed categorization. In this phase, subjects were instructed to categorize pictures on the basis of a taxonomic criterion, namely, the concept of life.

3) Presentation and recall of categorizable stimuli $B$. Using a second set of cards, the former procedure was repeated.

We expected that «practice» in categorization as an independent action in phase 2 would facilitate its subordination and inclusion into a more general action (memorization) in phase 3.

\section{RESULTS AND DISCUSSION}

\section{Directed categorization task}

In this task we evaluated subjects' clustering skills without being subordinate to a more general action. First, non-literate subjects obtained lower categorization scores than subjects in the higher levels (graduate and pregraduate). There were no significant differences between the performance of graduate and pregraduate groups. Subjects from non-literate group employed associative-contextual criteria for clustering stimuli, whereas subjects at higher levels tended to categorize stimuli on the basis of taxonomic criteria.

\section{Memory task}

In general, results obtained from the memory task were very similar to those obtained for the matching task: performance increases as school experience is longer. However, if we consider separately the amount of items recalled and clustered in recall, these general arguments must be modified.

The development of study actions was analyzed by comparing the two study periods. Non-literate subjects almost exclusively used examination actions, and very rarely used more complex actions. Subjects at a higher educational levels showed some interesting changes in their performance. In both the graduate and pregraduate groups the number of clustering actions increased from one period to the next, whereas the number of selfchecking actions decreased. The use of social support strategies did not differ between the three groups.

Results from the recall task varied depending on the index employed. There were significant differences in the number of items recalled between 
non-literate and the other two groups. These differences appear in both recall subtasks. If we focused on clusterings in the recall data, results were quite different. In the first subtask (A), differences appeared between the graduate group and the other groups, but there were no significant differences between pregraduate and non-literate groups. In the second subtask (B), after subjects had completed both the matching and recall A phases, results showed significant differences between pregraduate and non-literate levels, while graduate subjects mantained their superiority over the other two groups.

The pattern of performance is essentially the same in the two recall subtask for the three groups in qualitative terms with reported quantitative differences.

These results suggest that schooling influences the development of categorization and memory skills. Our interpretation focuses on the dynamic relationship between these skills, as well as on the way in which a cultural variable like schooling affects this relationship. A microgenetic analysis of subjects' performance provided evidence of qualitative changes in these interrelations, especially in the performance of subjects in the pre-graduate group. We maintain that these changes reflect the influence of formal education on cognitive development. The direction of this development seems oriented toward increasing a decontextualization of semiotic mediational means. 